\title{
Voltage assessment of distribution network with large penetration of PVs by probabilistic approach
}

\author{
T. Hiratsuka ${ }^{1}$, K. Nozaki ${ }^{1}$, M. Kato ${ }^{1}$ and Y. Hirata $^{2}$ \\ ${ }^{1}$ Department of Electrical and Electronic Engineering, Tokyo Denki University \\ 5 Senju asahi, Adachi, Tokyo, 120-8551, (Japan) \\ ${ }^{2}$ Institute of Industrial Science, The University of Tokyo, 4-6-1 Komaba, Meguro, Tokyo, 153-8505, (Japan)
}

\begin{abstract}
In this paper, the voltage profile in the Japanese distribution system with large penetration of photovoltaics (PVs) is estimated probabilistically by Monte-Carlo simulation. PV outputs and load demands are given as probability distributions with certain correlation factors.
\end{abstract}

\section{Key words}

photovoltaics, distribution network, voltage, Monte-Carlo simulation

\section{Introduction}

In recent years, issues of global warming and fossil fuel depletion have become serious, and PV attracts attention. When a large amount of PVs are introduced in the distribution network, the network voltage may rise by a reverse power flow. If the voltage deviates from the allowable voltage range (101 $\pm 6 \mathrm{~V}$ in Japan), generation opportunity of PV may be lost when a PV system is disconnected by PCS (Power Conditioning System) protection due to overvoltage.

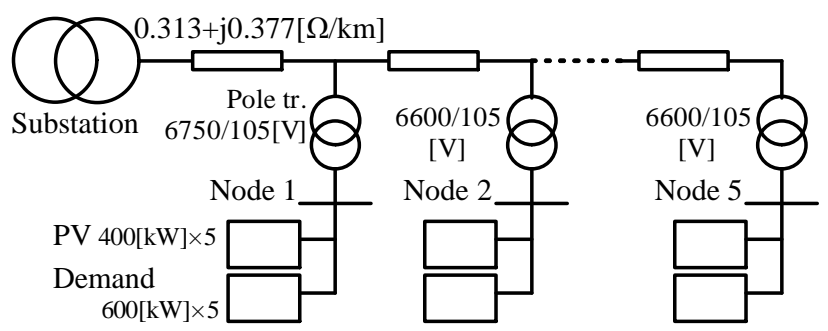

Fig.1. Distribution network

Table I. Setting of sending voltage

\begin{tabular}{|c|c|c|}
\hline \multicolumn{2}{|c|}{ Sending voltage } & \multirow{2}{*}{$\begin{array}{l}\text { Total demand - total PV output } \\
\qquad[\mathrm{kW}]\end{array}$} \\
\hline$[\mathrm{pu}]$ & [V] & \\
\hline 1.03 & 6,800 & 2,400 to 3,000 \\
\hline 1.015 & 6,700 & 1,500 to 2,400 \\
\hline 1 & 6,600 & 600 to 1,500 \\
\hline 0.985 & 6,500 & 600 and less \\
\hline
\end{tabular}

So far, the voltage profile of distribution network with PVs has been assessed only by the severest case that a large reverse power flow occurs, and not so many studies have explored probabilistic approaches. A probabilistic approach enables to efficiently install voltage controllers or storage batteries.

In this paper, the voltage in the distribution system with large penetration of PVs is estimated probabilistically by Monte-Carlo simulation. PV outputs and demands are given as random number sequences following the designated probability distribution with correlation factors.

\section{Simulation model}

\section{A. Distribution network model}

A distribution network in Japanese typical residential area is modelled with five sections. The distribution network model is shown in Fig. 1. The voltage is stepped down to $100 \mathrm{~V}$ from $6,600 \mathrm{~V}$ by a pole transformer installed at each five node, and it is supplied to customers. For protection from voltage deviation at lower limit in case that load is heavy, the tap ratio is set to lower at pole transformer where is far from the substation where network voltage is high. Otherwise, the tap ratio is set to higher at pole transformer where is near by the substation.

Load demand of $600 \mathrm{~kW}$ as its capacity and PV system of $400 \mathrm{~kW}$ as its capacity are connected in each node.

Sending voltage control scheme at the substation is generally performed in Japan. Therefore, voltage profile with sending voltage control is different from another voltage profile with sending voltage fixed as $1[\mathrm{pu}]$ $(6,600[\mathrm{~V}])$. The control scheme changes sending voltage by total demand. In this paper, total demand is subtracted by total PV output. Sending voltage with sending voltage control scheme is shown in Table I. 


\section{B. PV model}

The random number sequences which imitate PV outputs variation are generated. An appearance ratio distribution of PV output is based on the irradiation every hour during the daylight hours for every season obtained from irradiation data-base METPV-11 of NEDO (New Energy and Industrial Technology Development Organization). The correlation factor between each PV output is set to 1 because the studied area is assumed to be close.

\section{Demand model}

The appearance ratio distribution of demand variation is assumed to follow the Gamma distribution as like Fig. 2 from some Japanese data of a power company as like Fig. 3.

The difference of distribution of demand by seasons is defined by the average and standard deviation of demand shown in Table II.

As far as the correlation factor between PV output and demand, and the correlation factor between demands are concerned, very weak negative correlation is observed between PV output and demand in all seasons from limited data of PV output and demand. In winter, correlation factor between PV output and demand is lower than other seasons. On the other hand, demands have no correlation.

\section{Generation of random number sequence}

\section{A. Generation of random number sequence according to arbitrary distribution}

We used the following method to generate the random number sequence $y_{1}, y_{2}, \cdots \cdots, y_{N}$ ( $N$ : total simulation patterns) which imitates PV output variation:

The step function $f(x)$ in the interval $[0,1]$ like Fig. 4 is modeled on the density function of the actual PV appearance ratio distribution shown in Fig. 5. The interval $[0,1]$ is divided into $M$ (total section number) intervals by $x$ as follows:

$0=x_{1}<x_{2}<\cdots \cdots<x_{k}<x_{k+1}<\cdots \cdots<x_{M+1}=1$

The value of the $k$-th interval $\left[x_{k}, x_{k+1}\right]$ is set to $f_{k}(k=1,2, \cdots \cdots, M) . l_{1}$ which satisfies inequality (2) is found using the uniform random numbers $u_{1}, u_{2}, \cdots \cdots, u_{N}$ in interval $[0,1]$ :

$\sum_{k=1}^{l_{1}}\left(x_{k+1}-x_{k}\right) f_{k} \leq u_{1}<\sum_{k=1}^{l_{1}+1}\left(x_{k+1}-x_{k}\right) f_{k}$

Calculating the interval $\left[x_{l_{1}}, x_{l_{1}+1}\right]$ that satisfies inequality (2), we obtain

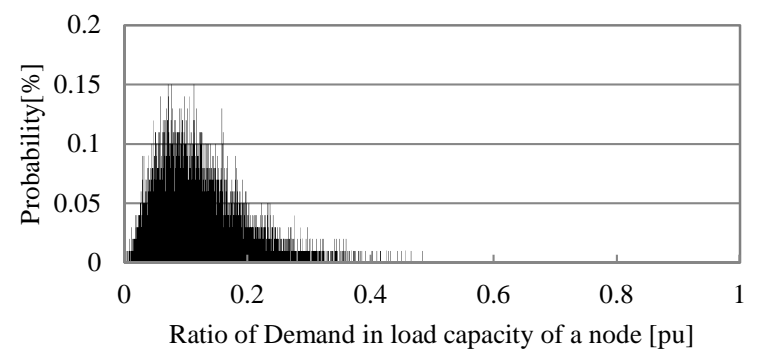

Fig.2. Profile of a random number sequence of the demand in spring

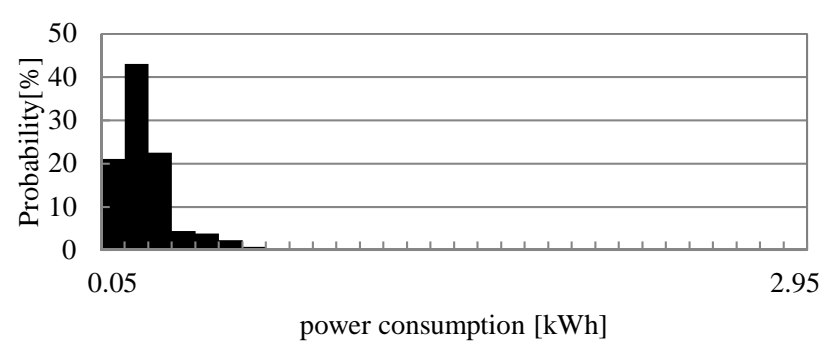

Fig.3. Demand profile of low voltage in spring

Table II. Average and standard deviation of demand in load capacity of a node [pu]

\begin{tabular}{|c|c|c|c|c|}
\hline & Spring & Summer & Autumn & Winter \\
\hline Average & 0.12 & 0.12 & 0.10 & 0.15 \\
\hline Standard deviation & 0.06 & 0.07 & 0.06 & 0.09 \\
\hline
\end{tabular}

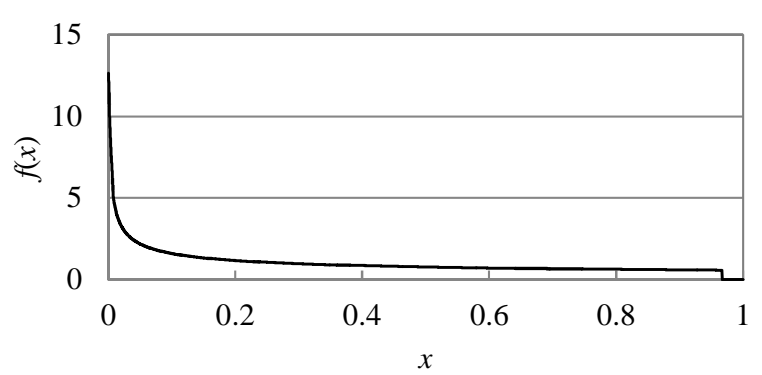

Fig.4. The step function $f(x)$ of solar irradiation in spring

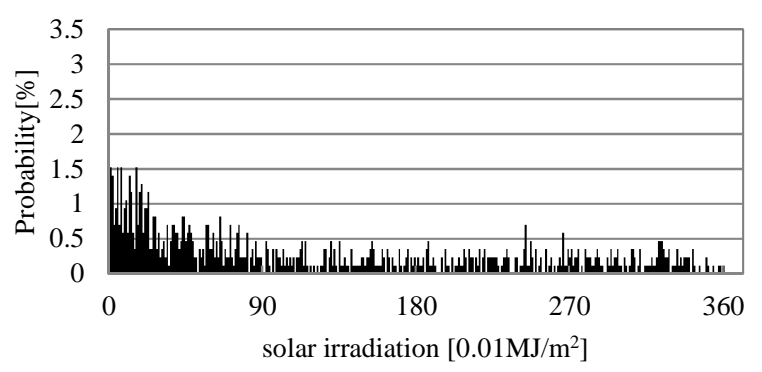

Fig.5. Actual solar irradiation profile in spring

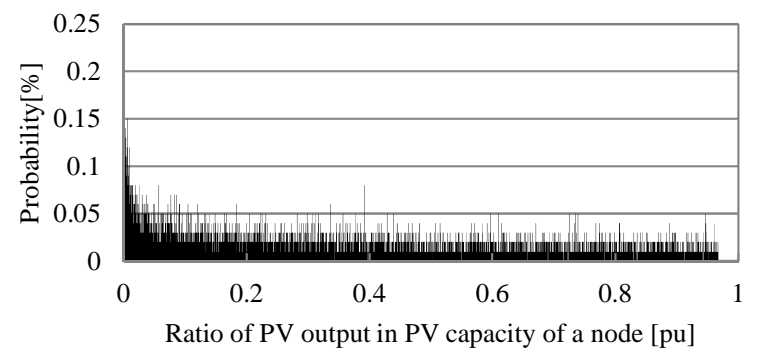

Fig.6. Example profile of a random number sequence of PV output in spring 


$$
y_{1}=\frac{x_{l_{1}}+x_{l_{1}+1}}{2}
$$

as the first random number. Similarly, random numbers $y_{2}, y_{3}, \cdots \cdots, y_{N}$ in interval $[0,1]$ are obtained. An example profile of a random number sequence of $\mathrm{PV}$ output is shown in Fig. 6.

\section{B. Generation of random number sequences according to arbitrary correlation matrices}

While the correlation factor between PV outputs is set to 1 for all nodes, correlation between PV output and demand at each node and correlation of demands may change with seasons. Therefore, the random numbers of each node demand are replaced in the following method until the current correlation matrix agrees with the target correlation matrix.

1) Set up the target correlation matrix between PV outputs and demands, and the target correlation matrix of demands as shown in Table III.

Table III. An example of correlation matrix of demands (correlation factor; 0.4)

Node 1
Node 2
Node 3
Node 4
Node 5 $\left[\begin{array}{ccccc}\text { Node 1 } & \text { Node 2 } & \text { Node 3 } & \text { Node 4 } & \text { Node 5 } \\ 1 & 0.4 & 0.4 & 0.4 & 0.4 \\ 0.4 & 1 & 0.4 & 0.4 & 0.4 \\ 0.4 & 0.4 & 1 & 0.4 & 0.4 \\ 0.4 & 0.4 & 0.4 & 1 & 0.4 \\ 0.4 & 0.4 & 0.4 & 0.4 & 1\end{array}\right]$

2) $y_{m}$ and $y_{n}$ in $y_{1}, y_{2}, \cdots \cdots, y_{N}$ of the random number sequence are exchanged.

Numbers $m$ and $n$ are random integers in [0, $N]$.

3) Let $p$ be a random number in $[0,1]$. If $p<\exp \left(-100 * c^{*} d\right)$, the state of being exchanged at 2) is kept. Otherwise, the previous state is kept. Here, $c$ is the number of exchange trials held until now. $d$ is defined as follow equality (4).

$$
\begin{aligned}
d= & \varepsilon_{P V \& \text { demand }}+\varepsilon_{\text {demands }} \\
& -\varepsilon_{P V \text { \&demand }} \min \\
& -\varepsilon_{\text {demands }_{\min }}
\end{aligned}
$$

Here, $\varepsilon_{P V \& \text { demand }}$ is total sum of square of the absolute value of each element of difference between current correlation matrix and target correlation matrix of PV and demand. $\varepsilon_{\text {demands }}$ is the same of demands. $\varepsilon_{P V \& \text { demand }_{\text {min }}}$ is the minimum of $\varepsilon_{P V \& \text { demand }}$ in trials until now. $\varepsilon_{\text {demand }_{\min }}$ and $\varepsilon_{P V \text { \&demand }_{\min }}$ are as well.

4) Repeat 2) and 3) until the current correlation matrix converges to the target one.

5) Replace the random numbers according to the procedures of 2) to 4) one by one also for another node demand distribution.

Example correlation diagrams of generated random number sequences are shown in Fig. 7 and Fig. 8.

\section{Simulation}

Power flows for the $N$ (here, $N$ is 10,000 ) combinations of PV output and demand generated by the above method are calculated to determine the voltage profile.

\section{A. The conditions of simulations}

Simulation conditions are summarised in Table IV. Power flows are simulated by each season (case 1, 4,
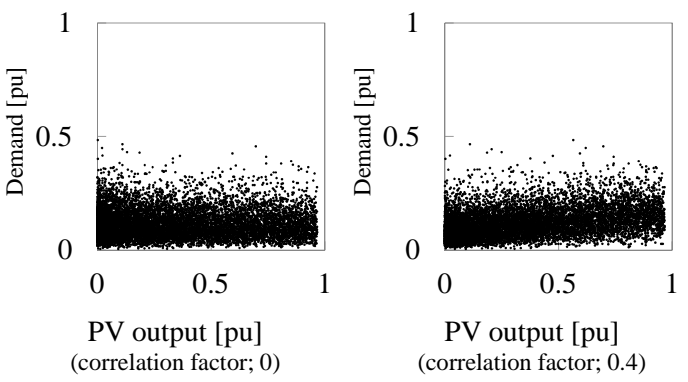

Fig.7. Correlation diagrams of PV output and demand in spring
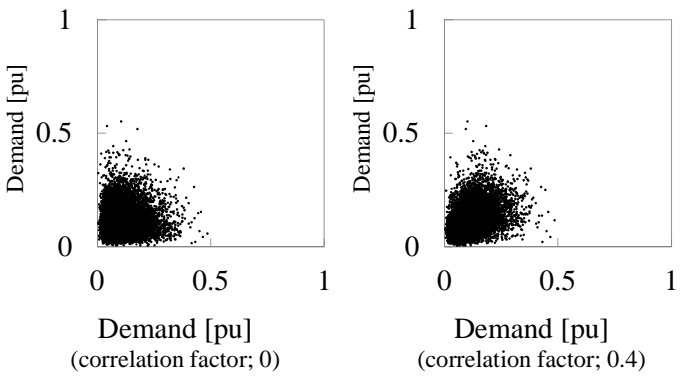

\begin{tabular}{|c|c|c|c|c|c|c|c|}
\hline \multirow[t]{2}{*}{ Case } & \multirow[t]{2}{*}{ Season } & \multicolumn{3}{|c|}{ Correlation factor } & \multicolumn{3}{|c|}{$\begin{array}{c}\text { Probability of voltage violation[\%] } \\
\text { sending voltage : controlled (constant) }\end{array}$} \\
\hline & & $\mathrm{PVs}$ & PV\&Demand & Demands & Node 3 & Node 4 & Node 5 \\
\hline 1 & \multirow{3}{*}{ Spring } & \multirow{3}{*}{1} & 0 & 0 & $0.0(12.6)$ & $0.0(18.5)$ & $0.2(20.6)$ \\
\hline 2 & & & 0.4 & 0 & $0.0(8.6)$ & $0.0(15.1)$ & $0.0(17.7)$ \\
\hline 3 & & & 0 & 0.4 & $0.0(12.7)$ & $0.0(18.6)$ & $0.6(20.8)$ \\
\hline 4 & Summer & 1 & 0 & 0 & $0.0(11.3)$ & $0.0(16.5)$ & $0.1(18.7)$ \\
\hline 5 & Autumn & 1 & 0 & 0 & $0.0(2.5)$ & $0.0(8.5)$ & $0.0(11.3)$ \\
\hline 6 & Winter & 1 & -0.2 & 0 & $0.0(6.2)$ & $0.0(10.4)$ & $0.0(12.5)$ \\
\hline
\end{tabular}

Fig.8. Correlation diagrams of demands in spring

Table IV. Probability of voltage violation in the distribution system 

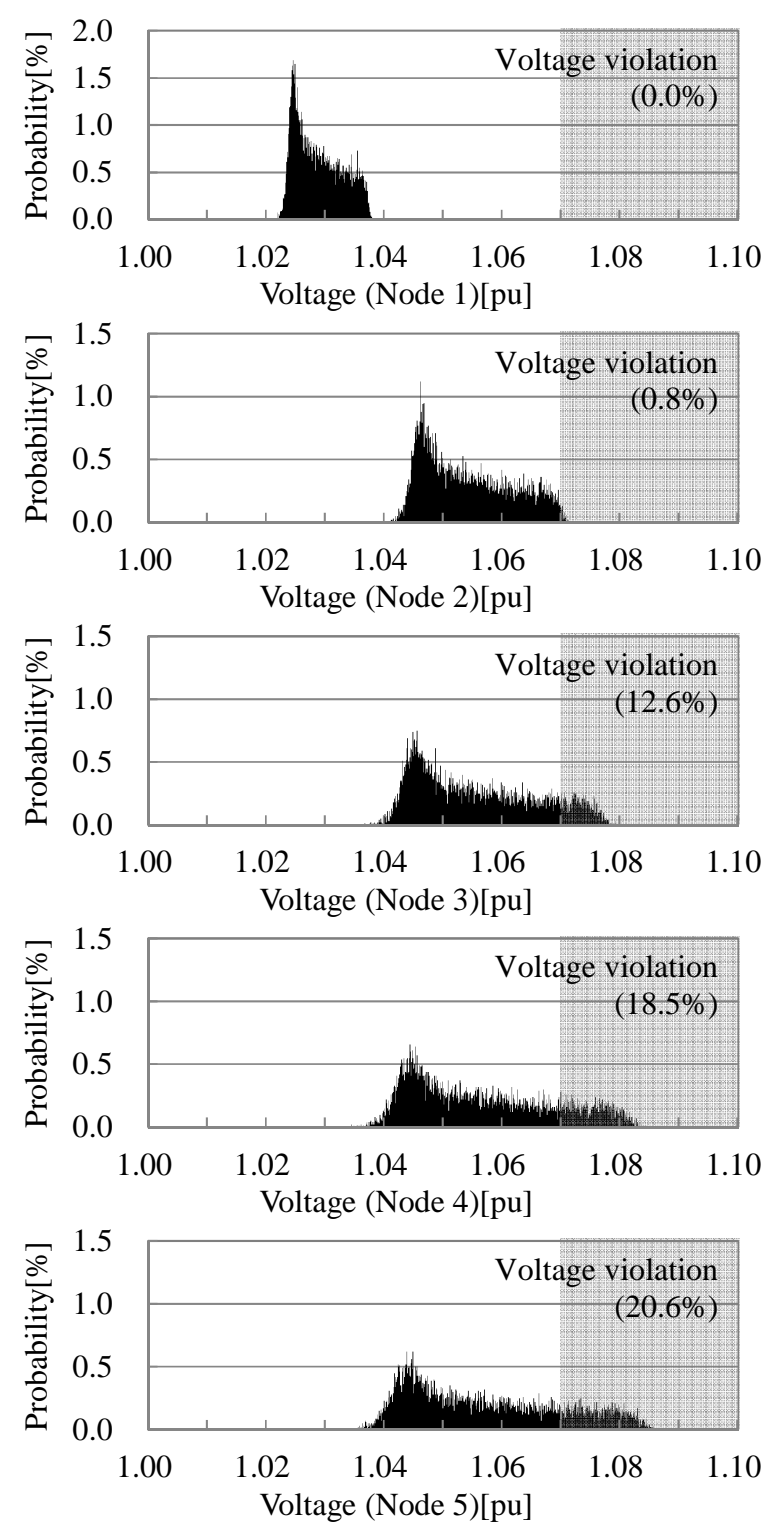

Fig.9. Voltage profile of case 1 with constant sending voltage

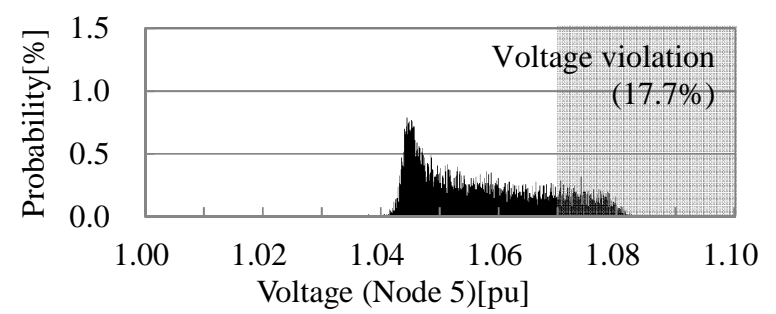

Fig.10. Voltage profile of case 2 with constant sending voltage

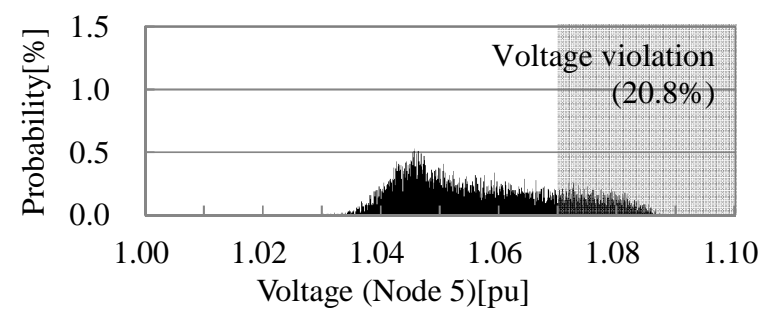

Fig.11. Voltage profile of case 3 with constant sending voltage

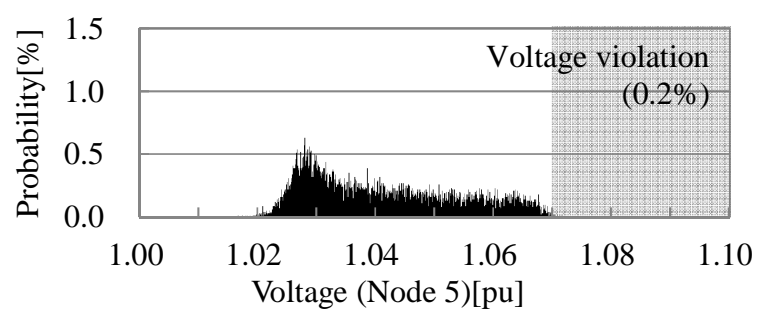

Fig.12. Voltage profile of case 1 with controlled sending voltage

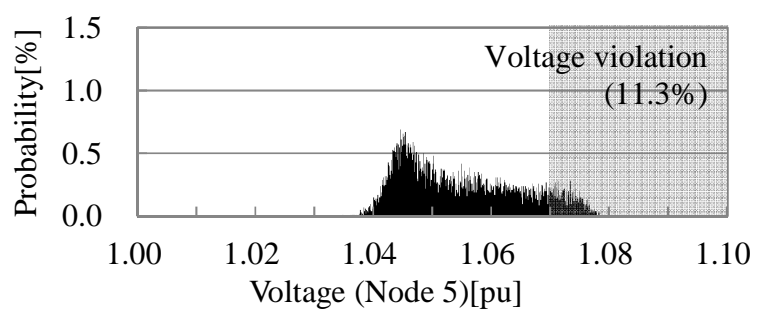

Fig.13. Voltage profile of case 5 with constant sending voltage

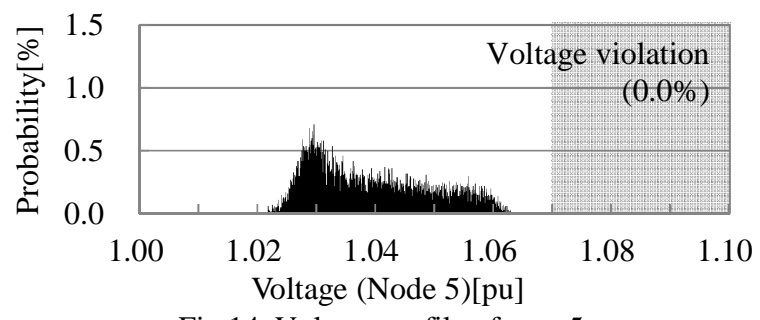

Fig.14. Voltage profile of case 5 with controlled sending voltage

5 , or 6) because combinations of PV output and demand are different in each season.

PV outputs are same in each node, so correlation factor of PVs is set to 1. Correlation factor of demands is set to 0 in all seasons expect winter. Only in winter, it is set to -0.2 . Furthermore, correlation between PV and demand is set to 0.4 in case 2 and between demands is set to 0.4 in case 3 in order to estimate effect to voltage profile of these factors change

\section{B. The results of simulations}

Simulation results are shown in Fig. 9 to 14 as well as in Table IV. Voltage violation occurs in the cases where PV output is high and demand is low. The violation probability is higher in spring than in summer since the $\mathrm{PV}$ output is almost the same and the demand is lower.

The farther from the substation, the more widely the voltage spreads. Therefore, voltage violation increases.

The higher the correlation factor between PV output and demand, the less the mismatch between PV output and demand becomes. Therefore, voltage violation decreases. On the other hand, the higher the correlation factor of demands, the more widely the demands spread. For this reason, voltage violation increases as well.

The sending voltage control scheme is very effective for decreasing the voltage violation probability. 


\section{Conclusion}

By giving PV outputs and demands as probability distributions, the voltage profile in the distribution network with large penetration of PVs is probabilistically evaluated.

Without degradation of power quality, a larger amount of PVs may be introduced by assessing PV-demand and demand-demand correlations probabilistically than the conventional study only considering the severest case.

Although the method of generating random number sequence with an arbitrary correlation factor is already known, there seems to be no stable method for generating the sequence of more than three dimensions. We need to grasp correlation factors more accurately to evaluate the profile more practically.

\section{Acknowledgement}

This research is partially supported by Aihara Project, the FIRST program from JSPS initiated by CSTP.

\section{References}

[1] Thomas Schreiber, Constrained Randomization of Time Series Data, Physical Review Letters 80, 2105-2108 (1998)

[2] K. Wakimoto, Knowledge of random numbers, Morikita publication, (1970), (in Japanese) 